\title{
Correlation Analysis of the Substituent Electronic Effects on the Allylic H-Abstraction in Cyclohexene by Phthalimide- $N$-oxyl Radicals: a DFT Study
}

\author{
Yong Sun, Wensong Zhang, Xingbang Hu, Haoran $\mathrm{Li}^{*}$
}

Department of Chemistry, Zhejiang University, Hangzhou 310027, P. R. China
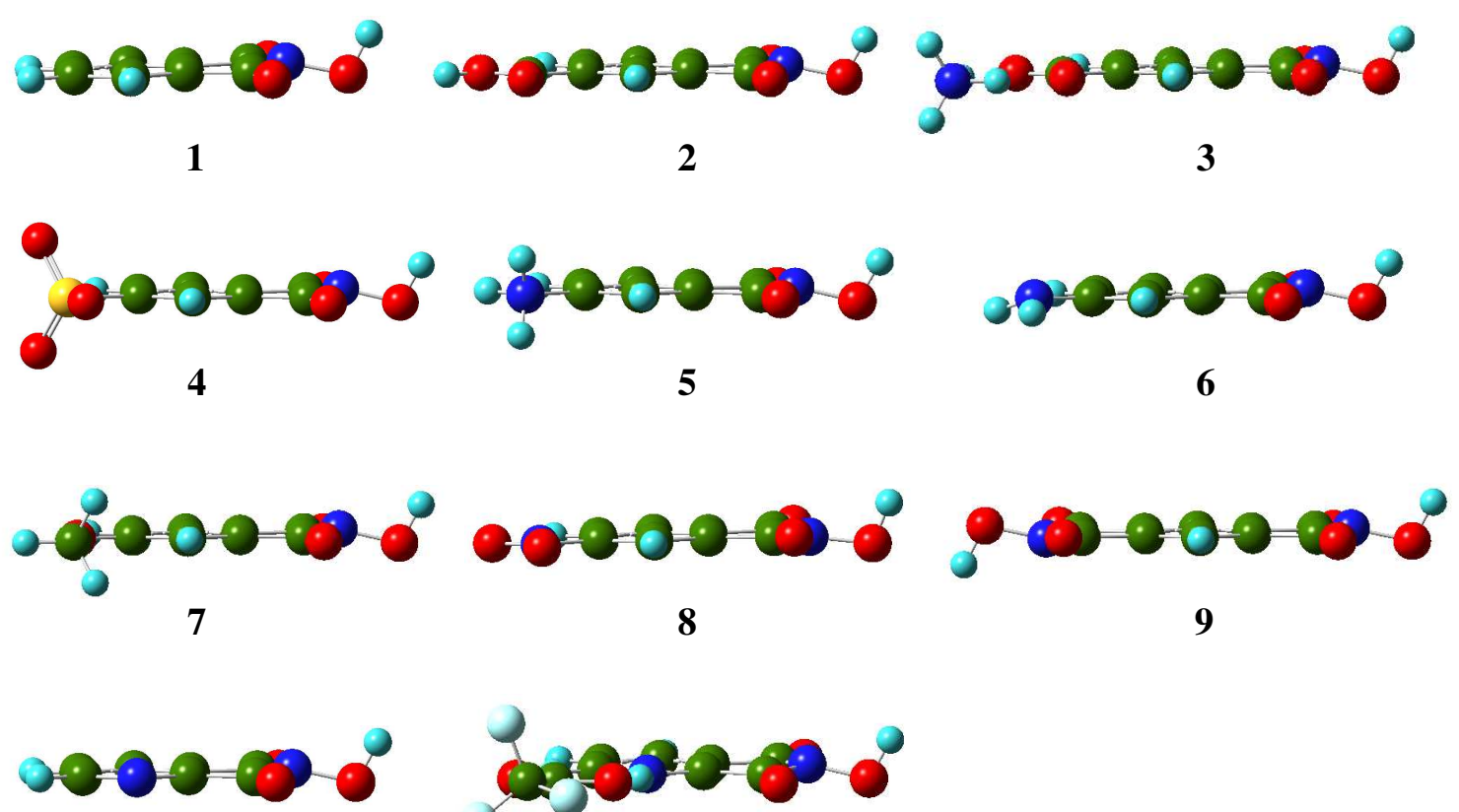

10

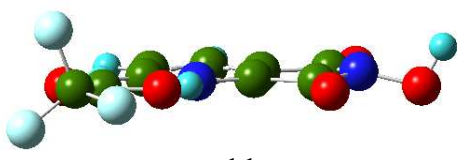

11

Figure S1 Optimized structures (side-view) for compounds 1 11. Definition of compounds 1 11 is shown in Figure 1.

${ }^{*}$ Corresponding author. Fax: +86-571-8795-1895

Email Address: lihr@zju.edu.cn (H. Li) 

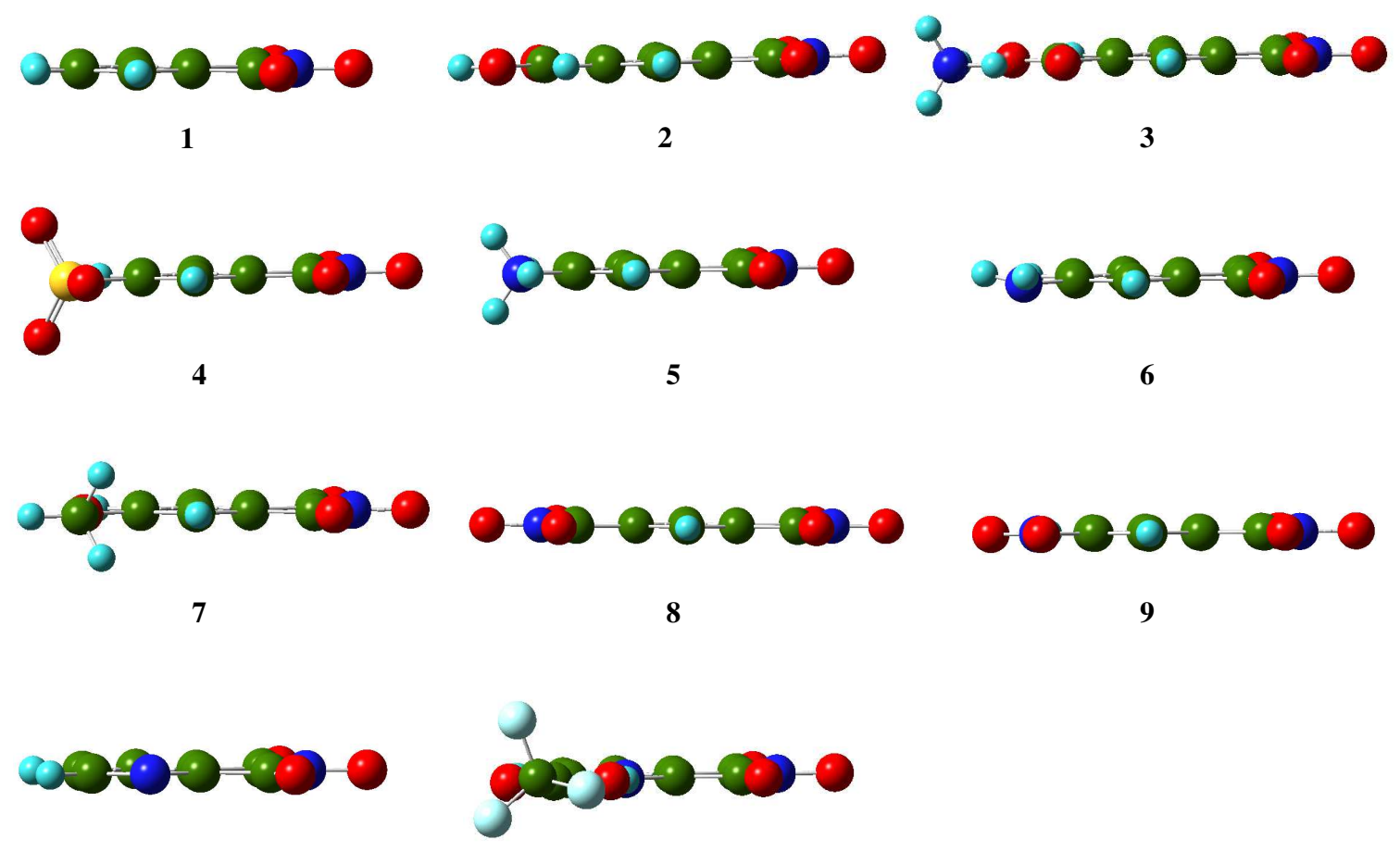

10

11

Figure S2 The optimized structures (side view) of the radicals corresponding to compounds 1 11. Definition of compounds 1 11 is shown in Figure 1 . 


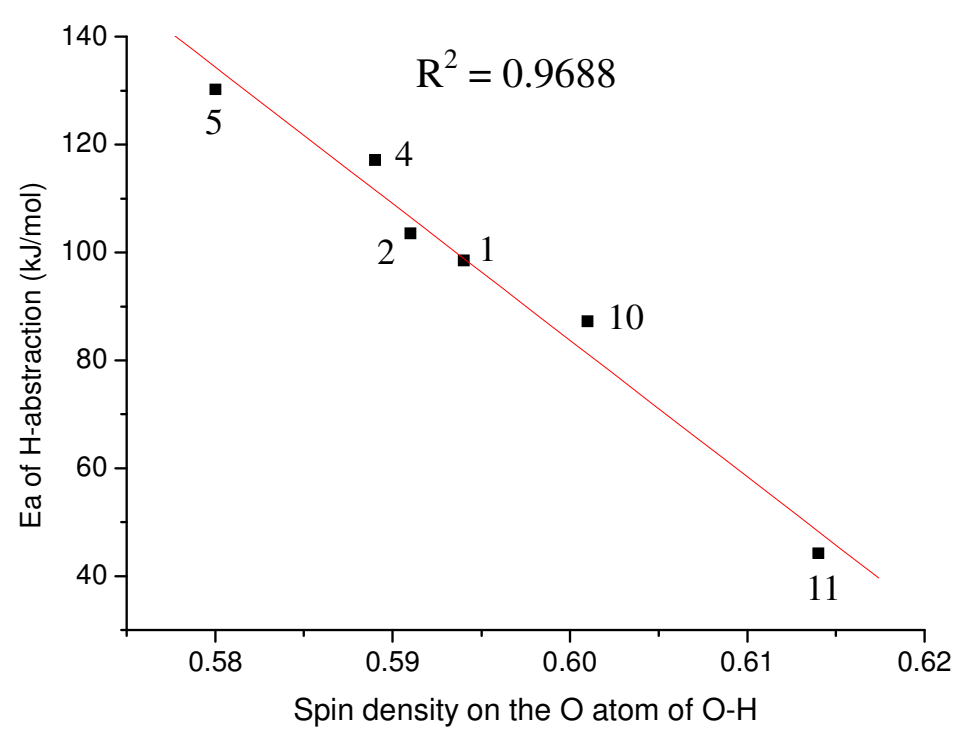

Figure S3 The allylic $\mathrm{H}$-abstraction activation energies as a function of the spin density of the active $\mathrm{O}$ atom, concerning the solvent effects using the default Gaussian 03 implementation of the polarizable continuum model (PCM). Definition of compounds 1 11 is shown in Figure 1. 


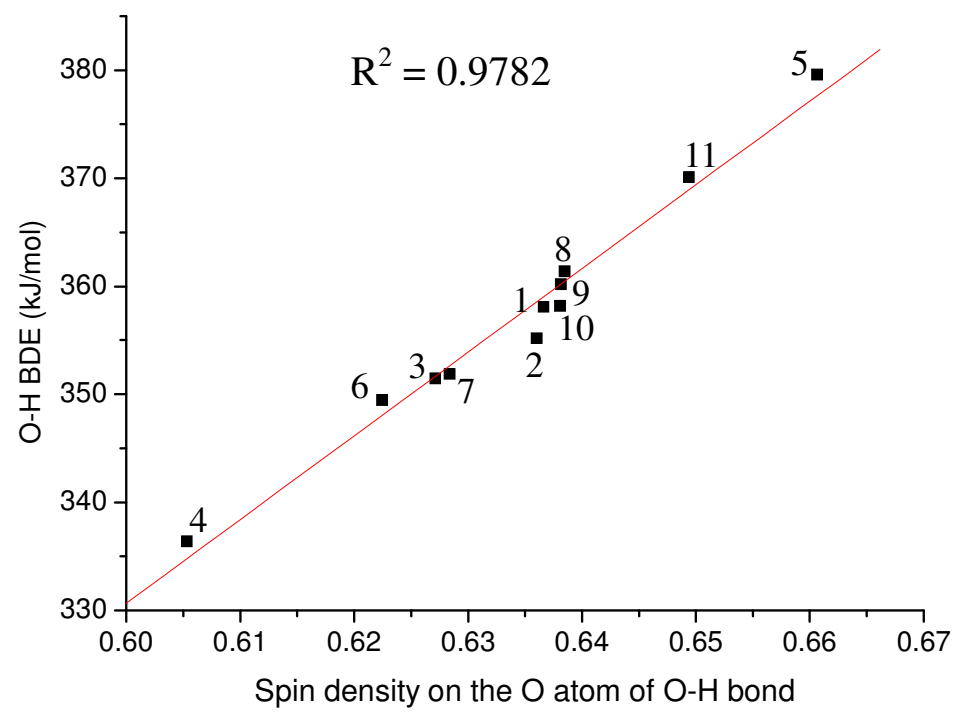

Figure S4 The bond dissociation energy (BDE) of O-H bond as a function of the spin density on the active $\mathrm{O}$ atom, using B3LYP/6-311++G** method. Definition of compounds $\mathbf{1} \sim \mathbf{1 1}$ is shown in Figure 1. 